TRANSACTIONS OF THE

AMERICAN MATHEMATICAL SOCIETY

Volume 358, Number 8, Pages 3697-3716

S 0002-9947(06)03856-6

Article electronically published on February 20, 2006

\title{
ON LINEAR TRANSFORMATIONS PRESERVING THE PÓLYA FREQUENCY PROPERTY
}

\author{
PETTER BRÄNDÉN
}

\begin{abstract}
We prove that certain linear operators preserve the Pólya frequency property and real-rootedness, and apply our results to settle some conjectures and open problems in combinatorics proposed by Bóna, Brenti and Reiner-Welker.
\end{abstract}

\section{INTRODUCTION}

Many sequences encountered in various areas of mathematics, statistics and computer science are known or conjectured to be unimodal or log-concave; see [7, 30, 32. A sufficient condition for a sequence to enjoy these properties is that it is a Pólya frequency ( $P F$ for short) sequence, or equivalently for finite sequences, that its generating function has only real and non-positive zeros. It is often the case that the generating function of a finite $P F$-sequence has more transparent properties when expanded in a basis other than the standard basis $\left\{x^{i}\right\}_{i>0}$ of $\mathbb{R}[x]$. Therefore it is natural to investigate how $P F$-sequences translate when expressed in various bases. This amounts to studying properties of the linear operator that maps one basis to another. A systematic study of this was first pursued by Brenti in [6]. This is also the theme of this paper.

In Section 3 we will study linear operators of the type

$$
\phi_{F}=\sum_{k=0}^{n} Q_{k}(x) \frac{d^{k}}{d x^{k}}
$$

where $F(x, z)=\sum_{k=0}^{n} Q_{k}(x) z^{k} \in \mathbb{R}[x, z]$. Here we will give sufficient conditions on $F$ for $\phi_{F}$ to preserve the $P F$-property. The results attained generalize and unify theorems of Hermite, Poulain, Pólya and Schur. In this section we will also give a sufficient condition for a family of natural $\mathbb{R}$-bilinear forms to preserve the $P F$-property in both arguments. This generalizes results of Wagner [10, 35, 36].

An important linear operator in combinatorics is the operator defined by $\mathcal{E}\left(\left(\begin{array}{l}x \\ i\end{array}\right)\right)$ $=x^{i}$, for all $i \in \mathbb{N}$. In Section 4 we will prove that whenever a polynomial $f$ of degree $d$ has non-negative coefficients when expanded in the basis $\left\{x^{i}(x+1)^{d-i}\right\}_{i=0}^{d}$, the polynomial $\mathcal{E}(f)$ will have only real, non-positive and simple zeros.

Received by the editors March 22, 2004 and, in revised form, September 14, 2004.

2000 Mathematics Subject Classification. Primary 05A15, 26C10; Secondary 05A19, 05A05, $20 \mathrm{~F} 55$.

This research was financed by the EC's IHRP Programme, within the Research Training Network "Algebraic Combinatorics in Europe", grant HPRN-CT-2001-00272, while the author was at Universitá di Roma "Tor Vergata", Rome, Italy.

(C)2006 American Mathematical Society Reverts to public domain 28 years from publication 
In the remainder of the paper we use the theory developed to settle some conjectures and open problems raised in combinatorics. In Section 5 we prove that the numbers $\left\{W_{t}(n, k)\right\}_{k=0}^{n-1}$ of $t$-stack sortable permutations in $\mathcal{S}_{n}$ with $k$ descents form $P F$-sequences when $t=2, n-2$, thereby settling two new cases of an open problem proposed by Bóna [2, 3 .

In Section 6 we prove that the $q$-Eulerian polynomials $A_{n}(x ; q)$, defined by Foata and Schützenberger [16] and further studied by Brenti in [9, have only real zeros for all integers $q$. This settles a conjecture raised by Brenti. Here we also continue the study of the $W$-Eulerian polynomials, defined for any finite Coxeter group $W$, and the $q$-analog $B_{n}(x ; q)$, initiated by Brenti in [8].

In Section 7 we prove that the $h$-vectors of a family of simplicial complexes associated to finite Weyl groups defined by Fomin and Zelevinski [17] are PF, thus settling an open problem raised by Reiner and Welker [28].

\section{NOTATION AND PRELIMINARIES}

In this section we collect definitions, notation and results that will be used frequently in the rest of the paper. Let $\left\{a_{i}\right\}_{i=0}^{\infty}$ be a sequence of real numbers. It is unimodal if there is a number $p$ such that $a_{0} \leq a_{1} \leq \cdots \leq a_{p} \geq a_{p+1} \geq \cdots$, and log-concave if $a_{i}^{2} \geq a_{i-1} a_{i+1}$ for all $i>0$.

An infinite matrix $A=\left(a_{i j}\right)_{i, j \geq 0}$ of real numbers is totally positive, $T P$, if all minors of $A$ are non-negative. An infinite sequence $\left\{a_{i}\right\}_{i=0}^{\infty}$ of real numbers is a Pólya frequency sequence (PF-sequence) if the matrix $\left(a_{i-j}\right)_{i, j \geq 0}$ is TP. Thus a PFsequence is by definition log-concave and therefore also unimodal. A finite sequence $a_{0}, a_{1}, a_{2}, \ldots, a_{n}$ is said to be $P F$ if the infinite sequence $a_{0}, a_{1}, a_{2}, \ldots, a_{n}, 0,0, \ldots$ is $P F$. A sequence $\left\{a_{i}\right\}_{i=0}^{\infty}$ is said to be $P F_{r}$ if all minors of size at most $r$ of $\left(a_{i-j}\right)_{i, j \geq 0}$ are non-negative. If the polynomials $\left\{b_{i}(x)\right\}_{i=0}^{d}$ are linearly independent over $\mathbb{R}$ and $r \in \mathbb{N}$ we define the set $P F_{r}\left[\left\{b_{i}(x)\right\}_{i=0}^{d}\right]$ to be

$$
P F_{r}\left[\left\{b_{i}(x)\right\}_{i=0}^{d}\right]=\left\{\sum_{i=0}^{d} \lambda_{i} b_{i}(x):\left\{\lambda_{i}\right\}_{i=0}^{\infty} \text { is } P F_{r}\right\},
$$

and $P F\left[\left\{b_{i}(x)\right\}_{i=0}^{d}\right]=\bigcap_{r=1}^{\infty} P F_{r}\left[\left\{b_{i}(x)\right\}_{i=0}^{d}\right]$.

The following theorem characterizes $P F$-sequences. It was conjectured by Schoenberg and proved by Edrei [15]; see also [22].

Theorem 2.1. Let $\left\{a_{i}\right\}_{i=0}^{\infty}$ be a sequence of real numbers with $a_{0}=1$. Then it is a PF-sequence if and only if the generating function can be expanded in a neighborhood of the origin as

$$
\sum_{i \geq 0} a_{i} z^{i}=e^{\gamma z} \frac{\prod_{i \geq 0}\left(1+\alpha_{i} z\right)}{\prod_{i \geq 0}\left(1-\beta_{i} z\right)}
$$

where $\gamma \geq 0, \alpha_{i}, \beta_{i}>0$ and $\sum_{i \geq 0}\left(\alpha_{i}+\beta_{i}\right)<\infty$.

A consequence of this theorem is that a finite sequence is $P F$ if and only if its generating function is a polynomial with only real non-positive zeros.

Let $f, g \in \mathbb{R}[x]$ be real-rooted with zeros: $\alpha_{1} \leq \cdots \leq \alpha_{i}$ and $\beta_{1} \leq \cdots \leq \beta_{j}$, respectively. We say that $f$ interlaces $g$, denoted $f \preceq g$, if $j=i+1$ and

$$
\beta_{1} \leq \alpha_{1} \leq \beta_{2} \leq \cdots \leq \beta_{j-1} \leq \alpha_{j-1} \leq \beta_{j} .
$$


We say that $f$ alternates left of $g$, denoted $f \ll g$, if $i=j$ and

$$
\alpha_{1} \leq \beta_{1} \leq \cdots \leq \beta_{i-1} \leq \alpha_{i} \leq \beta_{i} \text {. }
$$

If in addition $f$ and $g$ have no common zero, then we say that $f$ strictly interlaces $g$ and $f$ strictly alternates left of $g$, respectively. We also say that two polynomials $f$ and $g$ alternate if one of the polynomials alternates left of or interlaces the other. We will need two simple lemmata concerning these concepts. A polynomial is said to be standard if its leading coefficient is positive.

Lemma 2.2. Let $g$ and $\left\{f_{i}\right\}_{i=1}^{n}$ be real-rooted standard polynomials.

(i) If for each $1 \leq i \leq n$ we have either $g \ll f_{i}$ or $g \preceq f_{i}$. Then the sum $F=f_{1}+f_{2}+\cdots+f_{n}$ is real-rooted with $g \preceq F$ or $g \ll F$, depending on the degree of $F$.

(ii) If for each $1 \leq i \leq n$ we have either $f_{i} \ll g$ or $f_{i} \preceq g$. Then the sum $F=f_{1}+f_{2}+\cdots+f_{n}$ is real-rooted with $F \preceq g$ or $F \ll g$, depending on the degree of $F$.

Proof. The lemma follows easily by counting the sign-changes of $F$ at the zeros of g; see, e.g., [37, Prop. 3.5].

The next lemma is obvious.

Lemma 2.3. If $f_{0}, f_{1}, \ldots, f_{n}$ are real-rooted polynomials with $f_{0} \ll f_{n}$ and $f_{i-1} \ll$ $f_{i}$ for all $1 \leq i \leq n$, then $f_{i} \ll f_{j}$ for all $0 \leq i \leq j \leq n$.

The following theorem is a characterization of alternating polynomials due to Obreschkoff 24] and Dedieu [13.

Theorem 2.4. Let $f, g \in \mathbb{R}[x]$. Then $f$ and $g$ alternate (strictly alternate) if and only if all polynomials in the space

$$
\{\alpha f+\beta g: \alpha, \beta \in \mathbb{R}\}
$$

have only real (real and simple) zeros.

An immediate but non-trivial consequence of this theorem is

Corollary 2.5. Let $\phi: \mathbb{R}[x] \rightarrow \mathbb{R}[x]$ be a linear operator. Then $\phi$ preserves the real-rootedness property (real- and simple-rootedness property) only if $\phi$ preserves the alternating property (strictly alternating property).

We denote by $\mathbb{N}$ the set of natural numbers $\{0,1,2, \ldots\}$. The symmetric group of bijections $\pi:\{1,2, \ldots, n\} \rightarrow\{1,2, \ldots, n\}$ is denoted by $\mathcal{S}_{n}$. A descent in a permutation $\pi \in \mathcal{S}_{n}$ is an index $1 \leq i \leq n-1$ such that $\pi(i)>\pi(i+1)$. Let $\operatorname{des}(\pi)$ denote the number of descents in $\pi$. The Eulerian polynomials, $A_{n}(x)$, are defined by $A_{n}(x)=\sum_{\pi \in \mathcal{S}_{n}} x^{\operatorname{des}(\pi)+1}$ and satisfy (see e.g. [11])

$$
\sum_{k \geq 0} k^{n} x^{k}=\frac{A_{n}(x)}{(1-x)^{n+1}} .
$$

The binomial polynomials are defined by $\left(\begin{array}{l}x \\ 0\end{array}\right)=1$ and $\left(\begin{array}{l}x \\ k\end{array}\right)=\frac{x(x-1) \cdots(x-k+1)}{k !}$ for $k \geq 1$.

In several proofs we will implicitly use the fact that the zeros of a polynomial are continuous functions of the coefficients of the polynomial. In particular the limit of a sequence of real-rooted polynomials is again real-rooted. For a treatment of these matters we refer the reader to $[23]$. 


\section{A Class of linear operators preserving the $P F$-Property}

For any polynomial $F(x, z)=\sum_{k=0}^{n} Q_{k}(x) z^{k} \in \mathbb{R}[x, z]$ we define a linear operator $\phi_{F}: \mathbb{R}[x] \rightarrow \mathbb{R}[x]$ by

$$
\phi_{F}(f):=\sum_{k=0}^{n} Q_{k}(x) \frac{d^{k}}{d x^{k}} f(x) .
$$

In this section we will investigate for which $F \in \mathbb{R}[x, z]$ the linear operator $\phi_{F}$ preserves the real-rootedness and the $P F$-properties.

We will need some terminology and a theorem from $[5]$. For $\xi \in \mathbb{R}$ let $T_{\xi}$ : $\mathbb{R}[x] \rightarrow \mathbb{R}[x]$ be the translation operator defined by $T_{\xi}(f(x))=f(x+\xi)$. For any linear operator $\phi: \mathbb{R}[x] \rightarrow \mathbb{R}[x]$ we define a linear transform $\mathcal{L}_{\phi}: \mathbb{R}[x] \rightarrow \mathbb{R}[x, z]$ by

$$
\begin{aligned}
\mathcal{L}_{\phi}(f) & :=\phi\left(T_{z}(f)\right) \\
& =\sum_{n} \phi\left(f^{(n)}\right)(x) \frac{z^{n}}{n !} \\
& =\sum_{n} \frac{\phi\left(x^{n}\right)}{n !} f^{(n)}(z) .
\end{aligned}
$$

Definition 3.1. Let $\phi: \mathbb{R}[x] \rightarrow \mathbb{R}[x]$ be a linear operator. Define a function $d_{\phi}: \mathbb{R}[x] \rightarrow \mathbb{N} \cup\{-\infty\}$ by: If $\phi\left(f^{(n)}\right)=0$ for all $n \in \mathbb{N}$, we let $d_{\phi}(f)=-\infty$. Otherwise let $d_{\phi}(f)$ be the smallest integer $d$ such that $\phi\left(f^{(n)}\right)=0$ for all $n>d$. Hence $d_{\phi}(f) \leq \operatorname{deg} f$ for all $f \in \mathbb{R}[x]$.

The set $\mathcal{A}(\phi)$ is defined as follows: If $d_{\phi}(f) \in\{-\infty, 0\}$ and $\phi(f)$ is standard realand simple-rooted, then $f \in \mathcal{A}(\phi)$. Moreover, $f \in \mathcal{A}(\phi)$ if $d=d_{\phi}(f) \geq 1$ and all of the following conditions are satisfied:

(i) $\phi\left(f^{(i)}\right)$ all have leading coefficients of the same sign and $\operatorname{deg}\left(\phi\left(f^{(i-1)}\right)\right)=$ $\operatorname{deg}\left(\phi\left(f^{(i)}\right)\right)+1$ for $1 \leq i \leq d$,

(ii) $\phi(f)$ and $\phi\left(f^{\prime}\right)$ have no common real zero,

(iii) $\phi\left(f^{(d)}\right)$ strictly interlaces $\phi\left(f^{(d-1)}\right)$,

(iv) for all $\xi \in \mathbb{R}$ the polynomial $\mathcal{L}_{\phi}(f)(\xi, z)$ is real-rooted.

The following theorem is proved in [5].

Theorem 3.2. Let $\phi: \mathbb{R}[x] \rightarrow \mathbb{R}[x]$ be a linear operator. If $f \in \mathcal{A}(\phi)$, then $\phi(f)$ is real- and simple-rooted.

We will also need the following classical theorem of Hermite and Poulain. For a proof see [24].

Theorem 3.3. Let $f=a_{0}+a_{1} x+\cdots+a_{n} x^{n}$ and $g$ be real-rooted polynomials. Then the polynomial

$$
f\left(\frac{d}{d x}\right) g:=a_{0} g(x)+a_{1} g^{\prime}(x)+\cdots+a_{n} g^{(n)}(x)
$$

is real-rooted. Moreover, if $f\left(\frac{d}{d x}\right) g \neq 0$, then any multiple zero of $f\left(\frac{d}{d x}\right) g$ is a multiple zero of $g$.

The following theorem gives a sufficient condition for a polynomial to be mapped onto a real-rooted polynomial.

Theorem 3.4. Let $F=\sum_{k=0}^{n} Q_{k}(x) z^{k}$ be such that $Q_{0} \neq 0$ and

(I) For all $\xi \in \mathbb{R}, F(\xi, z)$ is real-rooted, 
(II) $Q_{0}$ strictly interlaces or strictly alternates left of $Q_{1}$, and $\operatorname{deg} Q_{0}=0$ or $Q_{0}$ and $Q_{1}$ have leading coefficients of the same sign.

Suppose that

(III) $f$ is real- and simple-rooted and that for $0 \leq k \leq \operatorname{deg} f$ the polynomials $\phi_{F}\left(f^{(k)}\right)$ have their leading term of the same sign with

$$
\operatorname{deg} \phi_{F}\left(f^{(k)}\right)=\operatorname{deg} Q_{0}+\operatorname{deg} f-k .
$$

Then $\phi_{F}(f)$ is real- and simple-rooted.

Proof. We will show that the set of real- and simple-rooted polynomials satisfying (III) is a subset of $\mathcal{A}\left(\phi_{F}\right)$ by verifying conditions (i)-(iv) of Definition 3.1. Condition (i) follows immediately from (III). For condition (iv) note that

$$
\mathcal{L}_{\phi}(f)(\xi, z)=\sum_{k=0}^{n} Q_{k}(\xi) f^{(k)}(\xi+z),
$$

so by Theorem 3.3 condition (iv) is satisfied. Suppose that $\eta$ is a common zero of $\phi_{F}(f)$ and $\phi_{F}\left(f^{\prime}\right)$. By (3.1) we have that 0 is a multiple zero of $\mathcal{L}_{\phi}(f)(\eta, z)$. Moreover, since $\mathcal{L}_{\phi}(f)(\eta, z)$ is not identically equal to zero, by (II), Theorem 3.3 tells us that 0 is a multiple zero of $f(\eta+z)$. This means that $\eta$ is a multiple zero of $f$ contrary to the assumption that $f$ is simple-rooted, and verifies condition (ii).

For condition (iii) we have to show that for all $\alpha \in \mathbb{R}$ such that $x+\alpha$ satisfies (III) the polynomial $\phi_{F}(1)=Q_{0}$ strictly interlaces $f(x):=\phi_{F}(x+\alpha)=(x+\alpha) Q_{0}+Q_{1}$. This follows from (II) when analyzing the sign of $f(x):=\phi_{F}(x+\alpha)$ at the zeros of $Q_{0}$ : Let $\alpha_{k}<\alpha_{k-1}<\cdots<\alpha_{1}$ be the zeros of $Q_{0}$ ordered by size. Suppose that $Q_{0}$ and $Q_{1}$ are standard and that $Q_{0}$ strictly interlaces or strictly alternates left of $Q_{1}$. Then $\operatorname{sgn} f\left(\alpha_{i}\right)=\operatorname{sgn} Q_{1}\left(\alpha_{i}\right)=(-1)^{i}$ for $1 \leq i \leq k$. By Rolle's theorem we know that $f$ has a zero in each interval $\left(\alpha_{i}, \alpha_{i+1}\right)$. This accounts for $k-1$ real zeros of $f$. Since $Q_{0}$ has positive sign, so does $f$ by condition (III). Now, because $f\left(\alpha_{1}\right)<0$ and $f$ is standard, $f$ must have a zero to the right of $\alpha_{1}$. We now know that $f$ has $k$ real zeros. The signs at $\alpha_{i}$ force the remaining zero to be in the interval $\left(-\infty, \alpha_{k}\right)$. Thus $Q_{0}$ strictly interlaces $f$ as was to be shown.

Now, if $Q_{0}=A \in \mathbb{R}$, then $\operatorname{deg} Q_{1} \leq 1$. Suppose that $Q_{1}=B \in \mathbb{R}$. Then clearly $A$ strictly interlaces $(x+\alpha) A+B$. If $Q_{0}=A$ and $Q_{1}=C x+D$ where $A, C, D \in \mathbb{R}$, then $f=(A+C) x+A \alpha+D$, so by (III) we have that $Q_{0}$ strictly interlaces $f$. This concludes the proof.

In some cases it may be convenient to have a sharper hypothesis. Therefore we state the following form of the theorem.

Corollary 3.5. Let $d \in \mathbb{N}$ be given and let $F=\sum_{k=0}^{n} Q_{k}(x) z^{k}$ be such that $Q_{0} \neq 0$ and

(i) For all $\xi \in \mathbb{R}, F(\xi, z)$ is real-rooted.

(ii) $Q_{0}$ strictly interlaces or strictly alternates left of $Q_{1}$, and $\operatorname{deg} Q_{0}=0$ or $Q_{0}$ and $Q_{1}$ have leading coefficients of the same sign.

(iii) The polynomials $\phi_{F}\left(x^{k}\right), 0 \leq k \leq d$ have the same sign and

$$
\operatorname{deg} \phi_{F}\left(x^{k}\right)=\operatorname{deg} Q_{0}+k .
$$

Then $\phi_{F}(f)$ is real-rooted (real- and simple-rooted) if $f$ is real-rooted (real and simple-rooted) and $\operatorname{deg}(f) \leq d$. 
Proof. The case of real- and simple-rooted $f$ follows immediately from Theorem 3.4 since (iii) implies (III). If $f$ is a real-rooted polynomial of degree at most $d$, then $f$ is the limit of a sequence $\left\{f_{k}\right\}_{k=0}^{\infty}$ of real- and simple-rooted polynomials of degree at most $d$. It follows that $\phi_{F}(f)$ is the limit of $\phi_{F}\left(f_{k}\right)$, and the thesis follows by continuity.

In the language of $P F$-sequences we have

Theorem 3.6. Let $d \in \mathbb{N}$ be given and let $F=\sum_{k=0}^{n} Q_{k}(x) z^{k} \in \mathbb{R}[x, z]$ be such that $Q_{0} \neq 0$ and

(i) For all $\xi \in \mathbb{R}, F(\xi, z)$ is real-rooted.

(ii) $\phi_{F}(1)$ strictly interlaces $\phi_{F}(x)$.

(iii) For all $0 \leq k \leq d$

$$
\operatorname{deg} \phi_{F}\left(x^{k}\right)=\operatorname{deg} Q_{0}+k,
$$

and $\phi_{F}\left(x^{k}\right) \in P F_{1}$.

Then $P F\left[\left\{\phi_{F}\left(x^{i}\right)\right\}_{i=0}^{d}\right] \subseteq P F\left[x^{i}\right]$.

Several old results can be derived from these last few theorems. In [25, p. 163] Pólya gave a theorem which he states was probably the most general theorem on real-rootedness known at the time. "Dieser Satz gehört wohl zu den allgemeinsten bekannten Sätzen über Wurzelrealität".

Theorem 3.7. Let $f(x)$ be a real-rooted polynomial of degree $n$, and let

$$
b_{0}+b_{1} x+\cdots+b_{n+m} x^{n+m} \quad(m \geq 0)
$$

be a real-rooted polynomial such that $b_{i}>0$ for $0 \leq i \leq n$. Then the equation

$$
G(x, y):=b_{0} f(y)+b_{1} x f^{\prime}(y)+b_{2} x^{2} f^{\prime \prime}(y)+\cdots+b_{n} x^{n} f^{(n)}(y)=0
$$

has $n$ real intersection points (counted with multiplicity) with the line

$$
s x-t y+u=0,
$$

provided that $s, t \geq 0, s+t>0$ and $u \in \mathbb{R}$.

Proof. We may assume that $s, t>0$ since the other cases follow by continuity when $s$ and/or $t$ tends to zero. Thus we may write the equation as

$$
a_{0} g(x)+a_{1} x g^{\prime}(x)+a_{2} x^{2} g^{\prime \prime}(x)+\cdots+a_{n} x^{n} g^{(n)}(x)=0,
$$

where $g(x)=f\left(s t^{-1} x+u t^{-1}\right)$ and $a_{i}=s^{i} t^{-i} b_{i}$. Now, we see that all hypotheses of Corollary 3.5 are satisfied for

$$
F(x, z)=a_{0}+a_{1} x z+a_{2} x^{2} z^{2}+\cdots+a_{n+m} x^{n+m} z^{n+m},
$$

when $d=n$.

We will later need one famous consequence of this theorem, $t=1, s=u=0$, due to Schur [29].

Theorem 3.8. Let $f=\sum_{k=0}^{n} a_{k} x^{k}$ and $g=\sum_{k=0}^{m} b_{k} x^{k}$ be two real-rooted polynomials such that $g$ has all zeros of the same sign. Then the polynomial

$$
(f S g)(x)=\sum_{k \geq 0}^{M} k ! a_{k} b_{k} x^{k},
$$

where $M=\min (m, n)$ has only real zeros. 
3.1. Multiplier-sequences. A multiplier-sequence is a sequence $T=\left\{\gamma_{i}\right\}_{i=0}^{\infty}$ of real numbers such that if a polynomial $f(x)=a_{0}+a_{1} x+\cdots+a_{n} x^{n}$ has only real zeros, then the polynomial

$$
T[f(x)]:=a_{0} \gamma_{0}+a_{1} \gamma_{1} x+\cdots+a_{n} \gamma_{n} x^{n}
$$

also has only real zeros. There is a characterization of multiplier-sequences due to Pólya and Schur [25, pp. 100-124], as follows.

Theorem 3.9. Let $T=\left\{\gamma_{i}\right\}_{i=0}^{\infty}$ be a sequence of non-negative real numbers and let $\phi(x)=T\left[e^{x}\right]=\sum_{k=0}^{\infty} \gamma_{k} \frac{x^{k}}{k !}$ be its exponential generating function. Then $T$ is a multiplier-sequence if and only if $\phi$ is a real entire function which can be written as

$$
\phi(x)=c x^{n} e^{\beta x} \prod_{k=1}^{\infty}\left(1+\delta_{k} x\right),
$$

where $c>0, \beta \geq 0, \delta_{k} \geq 0, n \in \mathbb{N}$ and $\sum_{k=1}^{\infty} \delta_{k}<\infty$.

The following lemma is well known (see [25]).

Lemma 3.10. A nonnegative multiplier-sequence has no internal zeros.

Theorem 3.11. Let $\left\{\lambda_{k}\right\}_{k=0}^{\infty}$ be a non-negative multiplier-sequence, and let $\alpha<$ $\beta \in \mathbb{R}$ be given. Define two $\mathbb{R}$-bilinear forms $\mathbb{R}[x] \times \mathbb{R}[x] \rightarrow \mathbb{R}[x]$ by

$$
\begin{aligned}
f \cdot g & :=\sum_{k \geq 0} \frac{\lambda_{k}}{k !} f^{(k)}(x) g^{(k)}(x)(x-\alpha)^{k}(x-\beta)^{k}, \\
f \circ g & :=\sum_{k \geq 0} \frac{\lambda_{k}}{k !} f^{(k)}(x) g^{(k)}(x)(x-\alpha)^{k} .
\end{aligned}
$$

If $f$ is real-rooted and $g$ is $[\alpha, \beta]$-rooted, then $f \cdot g$ is real-rooted. If $f$ is real-rooted and $g$ is $[-\infty, \alpha]$-rooted, then $f \circ g$ is real-rooted.

Proof. We prove the statement for $\cdot$ since the case for $\circ$ is similar. We may assume that $\lambda_{0}>0$. Clearly the theorem is true if $\lambda_{i}=0$ for all $i>0$, so by Lemma 3.10 we may assume that $\lambda_{1}>0$. Let $g$ have all zeros simple and in the interval $(\alpha, \beta)$, and let $\phi$ be the linear operator defined by $\phi(f)=f \cdot g$. Then $\phi=\phi_{F}$, where

$$
F(x, z)=\sum_{k \geq 0} \lambda_{k} \frac{g^{(k)}(x)}{k !}(x-\alpha)^{k}(x-\beta)^{k} z^{k} .
$$

Since $\left\{\lambda_{k}\right\}_{k \geq 0}$ is a multiplier sequence, $F(\xi, z)$ is real-rooted for all real choices of $\xi$. Now, $Q_{0}=\lambda_{0} g(x)$ and $Q_{1}=\lambda_{1}(x-\alpha)(x-\beta) g^{\prime}(x)$, so $Q_{0}$ strictly interlaces $Q_{1}$. Moreover, $\operatorname{deg} \phi\left(x^{k}\right)=\operatorname{deg} Q_{0}+k$ for all $k$, so all the hypothesis of Corollary 3.5 are fulfilled. Since any $[\alpha, \beta]$-rooted polynomial is the limit of polynomials which are $(\alpha, \beta)$ - and simple-rooted, the thesis follows by continuity.

There are a few bilinear forms on polynomials that occur frequently in combinatorics. Let $\#: \mathbb{R}[x] \times \mathbb{R}[x] \rightarrow \mathbb{R}[x]$ be defined by

$$
(f \# g)(x):=\sum_{k \geq 0} f^{(k)}(x) g^{(k)}(x) \frac{x^{k}}{k !} .
$$

This product is important when analyzing how the the zeros of $\sigma$-polynomials behave under disjoint union of graphs; see [10]. 
Theorem 3.12. Let $f$ be real-rooted and let $g$ have only real zeros of the same sign. Then $f \# g$ is real-rooted.

Proof. The theorem follows from Theorem 3.11, since $\{1\}_{k=0}^{\infty}$ is trivially a multipliersequence.

This generalizes a result of Wagner, who proved that $f \# g$ is real-rooted whenever $f$ and $g$ have only non-negative zeros; see [10, 35].

The diamond product of two polynomials $f$ and $g$ is given by

$$
(f \diamond g)(x)=\sum_{k \geq 0} \frac{f^{(k)}(x)}{k !} \frac{g^{(k)}(x)}{k !} x^{k}(x+1)^{k} .
$$

This product is important in the theory of $(P, \omega)$-partitions and the Neggers-Stanley conjecture and was first studied by Wagner in [36, 37]; see also Section 4 of this paper. Applying Theorem 3.11 with the multiplier-sequence $\left\{\frac{1}{k !}\right\}_{k \geq 0}$ we get

Theorem 3.13. Let $f$ be real-rooted and let $g$ have all zeros in the interval $[-1,0]$. Then $f \diamond g$ is real-rooted.

This was first proved by Wagner [37] under the additional hypothesis that $f$ has all zeros in $[-1,0]$, and generalized by the present author in [5].

A sequence of real numbers $\Gamma=\left\{\gamma_{k}\right\}_{k=0}^{\infty}$ is called a multiplier $n$-sequence if for any real-rooted polynomial $f=a_{0}+a_{1} x+\cdots+a_{n} x^{n}$ of degree at most $n$ the polynomial $\Gamma[f]:=a_{0} \gamma_{0}+a_{1} \gamma_{1} x+\cdots+a_{n} \gamma_{n} x^{n}$ is real-rooted. There is a simple algebraic characterization of multiplier $n$-sequences [12, as follows.

Theorem 3.14. Let $\Gamma=\left\{\gamma_{k}\right\}_{k=0}^{\infty}$ be a sequence of real numbers. Then $\Gamma$ is a multiplier n-sequence if and only if $\Gamma\left[(x+1)^{n}\right]$ is real-rooted with all its zeros of the same sign.

Recall the definition of the hypergeometric function ${ }_{2} F_{1}$ :

$$
{ }_{2} F_{1}(a, b ; c ; z)=\sum_{m=0}^{\infty} \frac{(a)_{m}(b)_{m} z^{m}}{(c)_{m} m !}
$$

where $(\alpha)_{0}=1$ and $(\alpha)_{m}=\alpha(\alpha+1) \cdots(\alpha+m-1)$ when $m \geq 1$. The Jacobi polynomial $P_{n}^{(\alpha, \beta)}(x)$ can be expressed as follows [26, p. 254]:

$$
P_{n}^{(\alpha, \beta)}(x)=\frac{(1+\alpha)_{n}}{n !}{ }_{2} F_{1}\left(-n, 1+\alpha+\beta+n ; 1+\alpha ; \frac{1-x}{2}\right) .
$$

We need the following lemma.

Lemma 3.15. Let $n$ be a positive integer and $r$ a non-negative real number. Then $\Gamma=\left\{\left(\begin{array}{c}-n-r \\ k\end{array}\right)\right\}_{k=0}^{\infty}$ is a multiplier n-sequence.

Proof. Let $r>0$. Then

$$
\begin{aligned}
\Gamma\left[(x+1)^{n}\right] & =\sum_{k=0}^{n}\left(\begin{array}{c}
-n-r \\
k
\end{array}\right)\left(\begin{array}{l}
n \\
k
\end{array}\right) x^{k} \\
& ={ }_{2} F_{1}(-n, n+r ; 1 ; x) \\
& =P_{n}^{(0, r-1)}(1-2 x),
\end{aligned}
$$

where the last equality follows from (3.3). Since the Jacobi polynomials are known (see [26]) to have all their zeros in $[-1,1]$ when $\alpha, \beta>-1$, we have that $\Gamma\left[(x+1)^{n}\right]$ 
has all its zeros in $[0,1]$. The case $r=0$ follows by continuity when we let $r$ tend to zero from above.

For any real number $q$ let $\Gamma_{q}:=\{q+k\}_{k=0}^{\infty}$. The following corollary was already known to Laguerre.

Corollary 3.16. Let $n>1$ be a positive integer. Then $\Gamma_{q}$ is a multiplier $n$-sequence if and only if $q \notin(-n, 0)$.

Proof. Let $q \in \mathbb{R}$ be given. We have to determine for which $n>1$ the zeros of $\Gamma_{q}\left[(x+1)^{n}\right]$ are all real and of the same sign. Now,

$$
\Gamma_{q}\left[(x+1)^{n}\right]=(x+1)^{n-1}\{(n+q) x+q\}
$$

and the theorem follows.

\section{The E-Transformation} by

The E-transformation is the invertible linear operator $\mathcal{E}: \mathbb{R}[x] \rightarrow \mathbb{R}[x]$ defined

$$
\mathcal{E}\left(\left(\begin{array}{l}
x \\
i
\end{array}\right)\right)=x^{i}
$$

for all $i \in \mathbb{N}$. The $P F$-preserving properties of this linear operator was first studied in [6] and later in [36, 37] and [5]. It is important in the theory of $(P, \omega)$-partitions since it maps the order-polynomial of a labeled poset to the $E$-polynomial of the same labeled poset; see [6, 36. In, 6] Brenti proved the following theorem. Let $\lambda(f)$ and $\Lambda(f)$ denote the smallest and the largest real zero of the polynomial $f$, respectively.

Theorem 4.1. Suppose that $f \in \mathbb{R}[x]$ has only real zeros and that $f(n)=0$ for all $n \in([\lambda(f),-1] \cup[0, \Lambda(f)]) \cap \mathbb{Z}$. Then $\mathcal{E}(f)$ has all zeros real and is non-positive.

In this section we will prove the following theorem.

Theorem 4.2. For all $n \in \mathbb{N}$ we have

$$
P F_{1}\left[\left\{x^{i}(x+1)^{n-i}\right\}_{i=0}^{n}\right] \subseteq P F\left[\left(\begin{array}{l}
x \\
i
\end{array}\right)\right] .
$$

Moreover if $f \in P F_{1}\left[\left\{x^{i}(x+1)^{n-i}\right\}_{i=0}^{n}\right]$, then $\mathcal{E}(f)$ has simple zeros and

$$
\mathcal{E}\left((x+1)^{d}\right) \ll \mathcal{E}(f) \ll \mathcal{E}\left(x^{d}\right) .
$$

The diamond product $(\underline{3.2})$ is intimately connected with the E-transformation. By the Vandermonde identity

$$
\left(\begin{array}{l}
x \\
i
\end{array}\right)\left(\begin{array}{l}
x \\
j
\end{array}\right)=\sum_{k \geq 0}\left(\begin{array}{c}
k \\
k-i, i+j-k, k-j
\end{array}\right)\left(\begin{array}{l}
x \\
k
\end{array}\right),
$$

it follows (see [37]) that

$$
(f \diamond g)(x):=\mathcal{E}\left(\mathcal{E}^{-1}(f) \mathcal{E}^{-1}(g)\right) .
$$

We will later need a symmetry property of $\mathcal{E}$. Let $\mathcal{R}: \mathbb{R}[x] \rightarrow \mathbb{R}[x]$ be the algebra automorphism defined by $\mathcal{R}(x)=-1-x$.

Lemma 4.3.

$$
\mathcal{R E}=\mathcal{E} \mathcal{R}
$$


Proof. Let $n$ be a non-negative integer. Using the identity

$$
\left(\begin{array}{c}
x+n \\
n
\end{array}\right)=\sum_{k=0}^{n}\left(\begin{array}{l}
n \\
k
\end{array}\right)\left(\begin{array}{l}
x \\
k
\end{array}\right)
$$

and the fact that $\left(\begin{array}{c}-x-1 \\ n\end{array}\right)=(-1)^{n}\left(\begin{array}{c}x+n \\ n\end{array}\right)$, we get

$$
\begin{aligned}
\mathcal{E R}\left(\begin{array}{l}
x \\
n
\end{array}\right) & =(-1)^{n} \mathcal{E}\left(\begin{array}{c}
x+n \\
n
\end{array}\right) \\
& =(-1)^{n} \sum_{k=0}^{n}\left(\begin{array}{l}
n \\
k
\end{array}\right) \mathcal{E}\left(\begin{array}{l}
x \\
k
\end{array}\right) \\
& =(-1-x)^{n} \\
& =\mathcal{R E}\left(\begin{array}{l}
x \\
n
\end{array}\right),
\end{aligned}
$$

and the lemma follows.

Lemma 4.4. Let $\alpha \in[-1,0]$ and let $f$ be a polynomial such that $\mathcal{E}(f)$ is $[-1,0]$ rooted. Then $\mathcal{E}((x-\alpha) f)$ is $[-1,0]$-rooted and $\mathcal{E}(f)$ interlaces $\mathcal{E}((x-\alpha) f)$. If $\mathcal{E}(f)$ in addition only has simple zeros, then so does $\mathcal{E}((x-\alpha) f)$.

Proof. Let $g=\mathcal{E}(f)$ and let $\alpha \in[-1,0]$. By (3.2) and (4.1) we have that

$$
\mathcal{E}((x-\alpha) f)=(x-\alpha) g+x(x+1) g^{\prime} .
$$

Since $g$ interlaces $(x-\alpha) g$ and $x(x+1) g^{\prime}$, it also interlaces the sum by Lemma 2.2 Also, if $x \notin[-1,0]$, then the summands have the same sign, so $\mathcal{E}((x-\alpha) f)$ cannot have any zeros outside $[-1,0]$. Suppose that $g$ has only simple zeros. Then by (4.2) the only possible common zeros of $g$ and $\mathcal{E}((x-\alpha) f)$ are 0 and -1 . If $\operatorname{deg}(f) \geq 1$ it also follows from (4.2) that the multiplicities of 0 and -1 of $\mathcal{E}((x-\alpha) f)$ are the same as those of $g$. Hence the (simple) zeros of $g$ separate the zeros of $\mathcal{E}((x-\alpha) f)$ except possibly at $0,-1$, and we conclude that $\mathcal{E}((x-\alpha) f)$ has only simple zeros.

Lemma 4.5. For all integers $n \geq 1$ we have

$$
(x+1) \mathcal{E}\left(x^{n}\right)=x \mathcal{E}\left((x+1)^{n}\right) .
$$

Proof. We may write

$$
x^{n}=\sum_{k=1}^{n} a_{k}\left(\begin{array}{l}
x \\
k
\end{array}\right),
$$

where $a_{k} \in \mathbb{R}$. Thus

$$
\begin{aligned}
\mathcal{E}\left((x+1)^{n}\right) & =\sum_{k=1}^{n} a_{k} \mathcal{E}\left[\left(\begin{array}{l}
x \\
k
\end{array}\right)+\left(\begin{array}{c}
x \\
k-1
\end{array}\right)\right] \\
& =\sum_{k=1}^{n} a_{k}\left(x^{k}+x^{k-1}\right) \\
& =(x+1) x^{-1} \mathcal{E}\left(x^{n}\right) .
\end{aligned}
$$

Let $f$ and $g$ be standard real-rooted polynomials of degree $n$ and let the zeros of $f$ and $g$ be $\alpha_{1} \leq \alpha_{2} \leq \cdots \leq \alpha_{n}$ and $\beta_{1} \leq \beta_{2} \leq \cdots \leq \beta_{n}$, respectively. We write $f \leq g$ if $\alpha_{i} \leq \beta_{i}$ for $1 \leq i \leq n$. 
Theorem 4.6. Suppose that $f$ and $g$ are $[-1,0]$-rooted with $f \leq g$. Then $\mathcal{E}(f)$ and $\mathcal{E}(g)$ are $[-1,0]$ - and simple-rooted, with $\mathcal{E}(f) \ll \mathcal{E}(g)$.

Proof. By Lemma 4.4 and induction we only have to show that $\mathcal{E}(f) \ll \mathcal{E}(g)$. If $f$ and $g$ have the same zeros except for one, i.e., $f=(x-\alpha) h$ and $g=(x-\beta) h$, where $\alpha<\beta$, then

$$
\mathcal{E}(g)=\mathcal{E}(f)-(\beta-\alpha) \mathcal{E}(h)
$$

and since $\mathcal{E}(h)$ interlaces $\mathcal{E}(f)$ we have $\mathcal{E}(f) \ll \mathcal{E}(g)$ by Lemma 2.2 ,

Now, suppose that $f$ and $g$ are $[-1,0]$-rooted polynomials of degree $n$ such that $f \leq g$. Then there are $[-1,0]$-rooted polynomials $\left\{h_{i}\right\}_{i=0}^{M}$ with

$$
(x+1)^{n}=h_{0} \leq h_{1} \leq \cdots \leq h_{M}=x^{n},
$$

such that $f, g \in\left\{h_{i}\right\}_{i=0}^{M}$ and $h_{i-1}$ and $h_{i}$ only differ in one zero for $1 \leq i \leq n$. We therefore have

$$
\mathcal{E}\left(h_{0}\right) \ll \mathcal{E}\left(h_{1}\right) \ll \cdots \ll \mathcal{E}\left(h_{M}\right),
$$

and since $\mathcal{E}\left(h_{0}\right) \ll \mathcal{E}\left(h_{M}\right)$, by Lemma 4.5 , the theorem follows from Lemma 2.3 ,

A consequence of Theorem 4.6 is that if $\left\{f_{i}\right\}_{i=1}^{m}$ is a sequence of standard [-1,0]rooted polynomials of the same degree $d$, then by Lemma 2.2] and Theorem 4.6, the image under $\mathcal{E}$ of any non-negative sum $F=\sum_{i=1}^{m} \mu_{i} f_{i}$ will be $[-1,0]$-rooted with

$$
\mathcal{E}\left((x+1)^{d}\right) \ll \mathcal{E}(F) \ll \mathcal{E}\left(x^{d}\right) .
$$

It is easy to see that a standard polynomial $f$ of degree $d$ is $[-1,0]$-rooted if and only if $f$ can be written as

$$
f(x)=(x+1)^{d} g\left(\frac{x}{x+1}\right),
$$

where $g$ is a standard and $(-\infty, 0)$-rooted. On the other hand, since $x^{i}(x+1)^{d-i}$ is $[-1,0]$-rooted, we have that $F$ can be written as a non-negative sum of standard $[-1,0]$-rooted polynomials of degree $d$ if and only if

$$
F(x)=\sum_{i=0}^{d} a_{i} x^{i}(x+1)^{d-i},
$$

where $a_{i} \geq 0$. This proves Theorem 4.2

\section{5. $t$-STACK SORTABLE PERMUTATIONS}

For relevant definitions regarding $t$-stack sortable permutations, we refer the reader to [2]. Let $W_{t}(n, k)$ be the number of $t$-stack sortable permutations in the symmetric group $\mathcal{S}_{n}$ with $k$ descents, and let

$$
W_{n, k}(x)=\sum_{k=0}^{n-1} W_{t}(n, k) x^{k} .
$$

Recently, Bóna [1, 3] showed that for fixed $n$ and $t$ the numbers $\left\{W_{t}(n, k)\right\}_{k=0}^{n-1}$ form a unimodal sequence. When $t=n-1$ and $t=1$ we get the Eulerian and the Narayana numbers (see [34] and [31, Exercise 6.36]), respectively. These are known to be $P F$-sequences, and Bóna [2, 3] has raised the question of whether this is true for general $t$. Here we will settle the problem to the affirmative for $t=2$ and $t=n-2$. 
The numbers $W_{2}(n, k)$ are surprisingly hard to determine despite their compact and simple form. It was recently shown that

$$
W_{2}(n, k)=\frac{(n+k) !(2 n-k-1) !}{(k+1) !(n-k) !(2 k+1) !(2 n-2 k-1) !} .
$$

See 4, 14, 19, 21] for proofs and more information on 2-stack sortable permutations.

From the case $r=0$ in Lemma 3.15 and the identity

$$
\sum_{k=0}^{n}\left(\begin{array}{c}
2 n-k-1 \\
n-1
\end{array}\right)\left(\begin{array}{l}
n \\
k
\end{array}\right) x^{k}=(-1)^{n} \sum_{k=0}^{n}\left(\begin{array}{c}
-n \\
k
\end{array}\right)\left(\begin{array}{l}
n \\
k
\end{array}\right)(-x)^{n-k}
$$

it follows that $\left(\begin{array}{c}2 n-k-1 \\ n-1\end{array}\right)$ is an $n$-sequence.

Theorem 5.1. For all $n \geq 0$ the sequence $\left\{W_{2}(n, k)\right\}_{k=0}^{n-1}$, which records 2-stack sortable permutations by descents, is PF.

Proof. We may write $W_{2}(n, k)$ as

$$
W_{2}(n, k)=\frac{\left(\begin{array}{c}
2 n-k-1 \\
n-1
\end{array}\right)\left(\begin{array}{c}
n+k \\
n-1
\end{array}\right)\left(\begin{array}{c}
2 n \\
2 k+1
\end{array}\right)}{n^{2}\left(\begin{array}{c}
2 n \\
n
\end{array}\right)} .
$$

A simple consequence of the notion of $P F$-sequences reads as follows: If $\left\{a_{i}\right\}_{i \geq 0}$ is $P F$, then so is $\left\{a_{k i}\right\}_{i \geq 0}$, where $k$ is any positive integer. Applying this to the polynomial $x(1+x)^{2 n}$ we see that $\sum_{k}\left(\begin{array}{c}2 n \\ 2 k+1\end{array}\right) x^{k}$ is real-rooted. Therefore the polynomial

$$
\sum_{k=0}^{n-1}\left(\begin{array}{c}
n+k \\
n-1
\end{array}\right)\left(\begin{array}{c}
2 n \\
2 k+1
\end{array}\right) x^{k}=\sum_{k=0}^{n-1}\left(\begin{array}{c}
2 n-k-1 \\
n-1
\end{array}\right)\left(\begin{array}{c}
2 n \\
2 k+1
\end{array}\right) x^{n-1-k}
$$

is real-rooted. Another application of Lemma 3.15 gives that $W_{n, 2}(x)$ is real-rooted.

It is easy to see that a permutation $\pi \in \mathcal{S}_{n}$ is $(n-2)$-stack sortable if and only if it is not of the form $\sigma n 1$. Thus the generating function satisfies

$$
x W_{n, n-2}(x)=A_{n}(x)-x A_{n-2}(x),
$$

where $A_{n}(x)$ is the $n$th Eulerian polynomial.

Theorem 5.2. For all real numbers $t>-2$ and integers $n>2$, the polynomial

$$
A_{n}(t, x)=A_{n}(x)+t x A_{n-2}(x)
$$

is real- and simple-rooted. Moreover, $A_{n}(t, x) / x$ strictly interlaces $A_{n+1}(t, x) / x$ for $-2<t \leq 3$.

Corollary 5.3. For all $n \geq 2$ we have that $\left\{W_{n-2}(n, k)\right\}_{k=0}^{n-1}$ is PF. Moreover, $W_{n, n-2}(x)$ strictly interlaces $W_{n+1, n-1}(x)$.

Proof of Theorem 5.2. It is well known that $A_{n-1}(x) \ll x A_{n-2}(x)$ and $A_{n-1}(x) \preceq$ $A_{n}(x)$. So by Lemma 2.2 we have that $A_{n}(t, x)$ is real- and simple-rooted for $t \geq 0$. However, when $t<0$ a similar argument does not apply.

Let $E_{n}(t, x)=A_{n}\left(t, \frac{x}{1+x}\right)$. Then

$$
E_{n}(t, x)=E_{n}(x)+t x(1+x) E_{n-2}(x),
$$


where the coefficient to $x^{k}$ in $E_{n}(x)$ counts the number of surjections $\sigma:[n] \rightarrow[k]$; see [6, 36]. These polynomials satisfy the recursion

$$
E_{n}(x)=x \frac{d}{d x}\left((1+x) E_{n-1}(x)\right),
$$

with initial condition $E_{1}(x)=x$. Thus, if we let $G_{n}(x)=E_{n+1}(x) / x$ we have the following recursion:

$$
G_{n}(x)=\frac{d}{d x}\left(x(1+x) G_{n-1}(x)\right),
$$

with $G_{0}(x)=1$. Obviously $G_{n}(x)$ is real- and simple-rooted. If we apply (5.1) two times we get the equation

$$
\begin{aligned}
G_{n}(x)=\left(1+6 x+6 x^{2}\right) G_{n-2}(x)+3 x(1+2 x)(1+x) G_{n-2}^{\prime}(x) & \\
& +x^{2}(1+x)^{2} G_{n-2}^{\prime \prime}(x),
\end{aligned}
$$

and for $G_{n}(t, x):=G_{n}(x)+t x(1+x) G_{n-2}(x)$ we have

$$
\begin{aligned}
G_{n}(t, x)=\left(1+(6+t) x+(6+t) x^{2}\right) G_{n-2}(x)+3 x(1+2 x) & (1+x) G_{n-2}^{\prime}(x) \\
& +x^{2}(1+x)^{2} G_{n-2}^{\prime \prime}(x) .
\end{aligned}
$$

To apply Theorem 3.4 we need show that for all $\xi \in \mathbb{R}$ and $-2<t<0$ the polynomial

$$
F(\xi, z):=\left(1+(6+t) \xi+(6+t) \xi^{2}\right)+3 \xi(1+2 \xi)(1+\xi) z+\xi^{2}(1+\xi)^{2} z^{2}
$$

is real-rooted. The discriminant of $F(\xi, z)$,

$$
\Delta(F(\xi, z))=\xi^{2}(1+\xi)^{2}\left(2+t+(3-t)(1+2 \xi)^{2}\right),
$$

is non-negative when $-2 \leq t \leq 3$, so $F(\xi, z)$ real-rooted for these $t$. Since all the $Q_{k}$ s are standard, it is easy to see that condition (III) in the statement of Theorem 3.4 is satisfied. Moreover, $1+(6+t) x+(6+t) x^{2}$ strictly interlaces $3 x(1+2 x)(1+x)$ when $t>-2$, so Theorem 3.4 applies. Since $G_{n}$ strictly interlaces $G_{n+1}$, we have by Theorem 3.4 and Corollary 2.5 that $\phi_{F}\left(G_{n}\right)$ strictly interlaces $\phi_{F}\left(G_{n+1}\right)$. Thus $A_{n}(t, x)$ strictly interlaces $A_{n+1}(t, x)$.

\section{6. $q$-EULERIAN AND $W$-EULERIAN POLYNOMIALS}

A $q$-analog of the Eulerian polynomials was introduced and studied in [16] and further studied in [9]. It is defined by

$$
A_{n}(x ; q):=\sum_{\pi \in \mathcal{S}_{n}} x^{\operatorname{exc}(\pi)} q^{c(\pi)},
$$

where $\mathrm{c}(\pi)$ and $\operatorname{exc}(\pi)$ denote the number of cycles and excedances in $\pi$, respectively. These polynomials satisfy the recursion

$$
A_{n+1}(x ; q)=(n x+q) A_{n}(x ; q)-x(x-1) \frac{\partial}{\partial x} A_{n}(x ; q),
$$

with initial condition $A_{0}(x ; q):=1$. See $[9$ for a proof. The following theorem appears in 9].

Theorem 6.1. Let $q \in \mathbb{R}, q>0$. Then the polynomials $A_{n}(x, q)$ have only real non-positive simple zeros.

Brenti also makes the following conjecture. 
Conjecture 6.2. Let $n, m \in \mathbb{N}$. Then $A_{n}(x ;-m)$ has only real zeros.

In what follows we will prove this conjecture using multiplier $n$-sequences. For $n \in \mathbb{N}$ define the polynomials $E_{n}(x ; q)$ by

$$
E_{n}(x ; q):=(1+x)^{n} A_{n}\left(\frac{x}{1+x} ; q\right) .
$$

It is clear that $E_{n}(x ; q)$ is real-rooted if and only if $A_{n}(x ; q)$ is real-rooted. These polynomials satisfy a somewhat easier recursion. Namely,

$$
E_{n+1}(x ; q)=(1+x)\left\{q E_{n}(x ; q)+x \frac{\partial}{\partial x} E_{n}(x ; q)\right\},
$$

with initial condition $E_{0}(x ; q)=1$. Now, for $q \in \mathbb{R}$ let $\Gamma_{q}: \mathbb{R}[x] \rightarrow \mathbb{R}[x]$ be the linear operator defined by $\Gamma_{q}(f(x))=q f(x)+x f^{\prime}(x)$. Since $\Gamma_{q}\left(x^{n}\right)=(q+n) x^{n}$ we may apply Corollary 3.16 .

Theorem 6.3. Let $q \in \mathbb{R}$ and $n \in \mathbb{N}$. If $q \geq 0, n \leq-q$ or $q \in \mathbb{Z}$, then $E_{n}(x ; q)$ has only real zeros.

Proof. We may write (6.1) as

$$
E_{n+1}(x ; q)=(x+1) \Gamma_{q}\left[E_{n}(x ; q)\right] .
$$

The cases $q \geq 0$ and $n \leq-q$ follow from Corollary 3.16 by induction. We may therefore assume that $q=-m$ is a negative integer. We claim that $\operatorname{deg} E_{n}(x ; q)=n$ if $n \leq m$ and $\operatorname{deg} E_{n}(x ; q)=m$ if $n \geq m$. From this the real-rootedness follows by Corollary 3.16 and induction. The case $n \leq m$ is clear since

$$
\Gamma_{q}\left[x^{n-1}\right]=-(m-n+1)<0 .
$$

The case $n>m$ also follows by induction. Suppose that $n \geq m$ and that $\operatorname{deg} E_{n}(x ; q)$ $=m$. Then by the recursion we have that $\operatorname{deg} E_{n+1}(x ; q) \leq m+1$. Moreover, since $\Gamma_{q}\left[x^{m}\right]=0$ we have that $\operatorname{deg} E_{n+1}(x ; q) \leq m$. Let $a \neq 0$ be the coefficient to $x^{m}$ of $E_{n}(x ; q)$. Then the coefficient to $x^{m}$ of $E_{n+1}(x ; q)$ is $a \Gamma_{q}\left[x^{m-1}\right]=-a$, so $\operatorname{deg} E_{n+1}(x ; q)=m$, and the thesis follows.

The Eulerian polynomial $P(W, x)$ of a finite Coxeter group $W$ is the polynomial

$$
P(W, x)=\sum_{\sigma \in W} x^{d_{W}(\sigma)},
$$

where $d_{W}(\sigma)$ is the number of $W$-descents of $\sigma$; see [8. This polynomial is also the generating function for the $h$-vector of the Coxeter complex associated to $(W, S)$. For Coxeter groups of type $A_{n}$ we have that $P\left(A_{n}, x\right)=A_{n}(x) / x$, the shifted Eulerian polynomial. Also, for Coxeter groups of type $B_{n}$ it is known (see [8] ) that $P\left(B_{n}, x\right)$, has only real zeros. It is easy to see that $P\left(W_{1} \times W_{2}, x\right)=$ $P\left(W_{1}, x\right) P\left(W_{2}, x\right)$ for finite Coxeter groups $W_{1}$ and $W_{2}$. Also, the real-rootedness can be checked ad hoc for the exceptional groups. Thus, by the classification of finite irreducible Coxeter groups, to prove that $P(W, x)$ has only real zeros for all finite Coxeter groups it suffices to prove that $P\left(D_{n}, x\right)$ is real-rooted for Coxeter groups of type $D_{n}$. The real-rootedness of $P\left(D_{n}, x\right)$ is conjectured by Brenti in [8]. It is known that the Eulerian polynomials of type $A_{n}, B_{n}$ and $D_{n}$ are related by (see [8, 27, 33])

$$
P\left(D_{n}, x\right)=P\left(B_{n}, x\right)-n 2^{n-1} x P\left(A_{n-1}, x\right) .
$$


This relationship was first noticed by Stembridge 33. One step towards proving the real-rootedness of $P\left(D_{n}, x\right)$ is to learn more about the relationships between the zeros of $P\left(B_{n}, x\right)$ and $P\left(A_{n}, x\right)$.

Brenti [8] introduced a $q$-analog of $P\left(B_{n}, x\right)$

$$
B_{n}(x ; q)=\sum_{\sigma \in B_{n}} q^{N(\sigma)} x^{d_{B}(\sigma)}
$$

where $d_{B}(\sigma)$ is the number of $B_{n}$-descents of $\sigma$ and $N(\sigma)$ is the number of negative entries of $\sigma$; see 8 . He proved that

$$
\sum_{i \geq 0}((1+q) i+1)^{n} x^{i}=\frac{B_{n}(x ; q)}{(1-x)^{n+1}},
$$

and that $B_{n}(x ; q)$ is real- and simple-rooted for all $q \geq 0$. Suppose that $f(i)$ is a polynomial in $i$ of degree $d$; then the polynomial $W(f)$ is defined by

$$
\sum_{i \geq 0} f(i) x^{i}=\frac{W(f)(x)}{(1-x)^{d+1}}
$$

One can show (see [6]) that $\mathcal{E}(f)$ and $W(f)$ are related by

$$
\mathcal{E}(f)(x)=(1+x)^{\operatorname{deg}(f)} W(f)\left(\frac{x}{1+x}\right) .
$$

It follows that $W(f)$ has only real non-positive roots if and only if $\mathcal{E}(f)$ is $[-1,0]$ rooted.

Since $((1+q) i+1)^{n}$ is a $[-1,0]$-rooted polynomial in $i$ for any $q \geq 0$, it follows from, e.g. Theorem 4.2 , that $B_{n}(x ; q)$ is real-rooted in $x$ for any fixed $q \geq 0$. It is natural to generalize $B_{n}(x ; q)$ to have $n+1$ parameters as $B_{n}(x ; \mathbf{q}):=$ $W\left(\prod_{i=0}^{n}\left(\left(1+q_{i}\right) x+1\right)\right)$. This polynomial has a nice combinatorial interpretation, as follows.

Theorem 6.4. For all $n \in \mathbb{N}$ we have

$$
B_{n}(x, \mathbf{q})=\sum_{\sigma \in B_{n}} q_{1}^{\chi_{1}(\sigma)} q_{2}^{\chi_{2}(\sigma)} \cdots q_{n}^{\chi_{n}(\sigma)} t^{d_{B}(\sigma)}
$$

where

$$
\chi_{i}(\sigma)= \begin{cases}1 & \text { if } \sigma_{i}<0 \\ 0 & \text { if } \sigma_{i}>0\end{cases}
$$

Proof. The proof is an obvious generalization of the proof of Theorem 3.4 of [8].

Note that this theorem gives a semi-combinatorial interpretation of the $W$ transform of any $[-1,0)$-rooted polynomial.

Corollary 6.5. Let $n \in \mathbb{N}$ and let $q_{1}, q_{2}, \ldots, q_{n}$ be non-negative real numbers. Then $B_{n}(x ; \mathbf{q})$ has only real and simple zeros.

We need the following lemma on the degree of $W(f)$.

Lemma 6.6. Let $f \in \mathbb{R}[x]$. Then

$$
\operatorname{deg} W(f)=\operatorname{deg} f-\operatorname{mult}(-1, \mathcal{E}(f)) .
$$

Moreover, mult $(-1, \mathcal{E}(f))$ is equal to the maximal integer $k$ such that

$$
(x+1)(x+2) \cdots(x+k)
$$

divides $f$. 
Proof. Since $\operatorname{deg} \mathcal{E}(f)=\operatorname{deg} f$ for all $f$, we have by (6.4) that $\operatorname{deg} W(f)=\operatorname{deg} f-$ $\operatorname{mult}(-1, \mathcal{E}(f))$. If we expand $f$ in the basis $\left\{\left(\begin{array}{c}-x-1 \\ i\end{array}\right)\right\}$ as

$$
\begin{aligned}
f(x) & =\sum_{i \geq 0}(-1)^{i} a_{i}\left(\begin{array}{c}
-x-1 \\
i
\end{array}\right) \\
& =\sum_{i \geq 0} \frac{a_{i}}{i !}(x+1) \cdots(x+i),
\end{aligned}
$$

we have by Lemma 4.3 that

$$
\mathcal{E}(f)(x)=\sum_{i \geq 0} a_{i}(x+1)^{i}
$$

and the lemma follows.

We now have more precise knowledge of the location of the zeros of $B_{n}(x ; q)$ for any given $q \geq 0$.

Theorem 6.7. Let $0<q<t \in \mathbb{R}$ and let $n>0$ be an integer. Then

$$
B_{n}(x ; 0) \preceq B_{n}(x ; t) \ll B_{n}(x ; q) \ll x B_{n}(x ; 0),
$$

where the first three polynomials have no common zeros.

Proof. Let $0<r<s<1$. Then by the proof of Lemma 4.4 we have

$$
\begin{aligned}
\mathcal{E}\left(x^{n}\right) \ll \mathcal{E}\left(x(x+r)^{n-1}\right) & \ll_{\text {strict }} \mathcal{E}\left((x+r)^{n}\right) \ll_{\text {strict }} \mathcal{E}\left((x+r)^{n-1}(x+s)\right) \\
\ll \mathcal{E}\left((x+s)^{n}\right) \ll_{\text {strict }} \mathcal{E}\left((x+s)^{n-1}(x+1)\right) & \ll \mathcal{E}\left((x+1)^{n}\right),
\end{aligned}
$$

where $\ll_{\text {strict }}$ means strictly alternating left of. Since $(x+1) \mathcal{E}\left(x^{n}\right)=x \mathcal{E}\left((x+1)^{n}\right)$, this implies

$$
\mathcal{E}\left(x^{n}\right) \ll_{\text {strict }} \mathcal{E}\left((x+r)^{n}\right) \ll_{\text {strict }} \mathcal{E}\left((x+s)^{n}\right) \ll_{\text {strict }} \mathcal{E}\left((x+1)^{n}\right) .
$$

Now since

$$
B_{n}(x ; q)=(q+1)^{n} W\left(\left(x+\frac{1}{1+q}\right)^{n}\right)=(q+1)^{n}(1-x)^{n} \mathcal{E}\left(\left(x+\frac{1}{1+q}\right)^{n}\right)\left(\frac{x}{1-x}\right),
$$

we see by Lemma 6.6 that $\operatorname{deg} B_{n}(x ; 0)=n-1$ and $\operatorname{deg} B_{n}(x ; q)=n$ if $q \neq 0$. Moreover, the alternating property is preserved under the operation (6.4), and the theorem follows.

It follows from (6.2) that $P\left(B_{n}, x\right)=B_{n}(x ; 1)$ and $P\left(A_{n}, x\right)=B_{n}(x ; 0)$.

Corollary 6.8. For all integers $n \geq 1$ we have that $P\left(A_{n}, x\right)$ strictly interlaces $P\left(B_{n}, x\right)$.

Since $P\left(A_{n}, x\right) \ll x P\left(A_{n-1}, x\right)$ and $P\left(A_{n}, x\right) \preceq P\left(B_{n}, x\right)$, we have by Lemma 2.2 that for all $t \geq 0$ the polynomial $P\left(B_{n}, x\right)+t x P\left(A_{n-1}, x\right)$ is real-rooted. Unfortunately a similar argument does not apply when $t<0$.

One can extract more from (6.3). Brenti [8] proved that the polynomial

$$
\sum_{\sigma \in B_{n}, N(\sigma) \in\{k, n-k\}} x^{d_{B}(\sigma)}
$$

is real-rooted for all choices of $0 \leq k \leq n$. Using Theorem 4.6 we can extend this result to the following corollary. 
Corollary 6.9. Let $S$ be any subset of $[0, n]$. Then the polynomial

$$
P\left(B_{n}, S ; x\right):=\sum_{\sigma \in B_{n}, N(\sigma) \in S} x^{d_{B}(\sigma)}
$$

has only real and simple zeros.

Proof. Comparing the coefficient of $q^{i}$ in both sides of (6.3) we see that $P\left(B_{n}, S ; x\right)$ $=W\left(f_{n}(S ; x)\right)$, where

$$
f_{n}(S ; x)=\sum_{s \in S}\left(\begin{array}{l}
n \\
s
\end{array}\right) x^{s}(x+1)^{n-s} .
$$

So the theorem follows from Theorem 4.2 .

One instance of Corollary 6.9 is particularly interesting. Recall that a Coxeter group of type $D_{n}$ is isomorphic to the subgroup

$$
D_{n}=\left\{\sigma \in B_{n}: 2 \mid N(\sigma)\right\} .
$$

Hence, we have the following corollary.

Corollary 6.10. For all $n \in \mathbb{N}$ the polynomial

$$
\sum_{\sigma \in D_{n}} x^{d_{B}(\sigma)}
$$

has only real and simple zeros.

Note that the above polynomial is not $P\left(D_{n}, x\right)$, since $B_{n^{-}}$-descents and $D_{n^{-}}$ descents are not the same.

\section{The $h$-VeCtor of A FAMILY of SIMPLicial COMPlEXeS DEFINED BY FOMIN AND ZELEVINSKY}

Fomin and Zelevinsky [17] recently associated to any finite Weyl group $W$ a simplicial complex $\Delta_{F Z}(W)$. For the classical Weyl groups the corresponding $h$ vectors (polynomials) are given by

$$
\begin{aligned}
h\left(\Delta_{F Z}\left(A_{n-1}\right), x\right) & =\frac{1}{n} \sum_{k=0}^{n-1}\left(\begin{array}{l}
n \\
k
\end{array}\right)\left(\begin{array}{c}
n \\
k+1
\end{array}\right) x^{k}, \\
h\left(\Delta_{F Z}\left(B_{n}\right), x\right) & =\sum_{k=0}^{n}\left(\begin{array}{l}
n \\
k
\end{array}\right)\left(\begin{array}{l}
n \\
k
\end{array}\right) x^{k}, \\
h\left(\Delta_{F Z}\left(D_{n}\right), x\right) & =h\left(\Delta_{F Z}\left(B_{n}\right), x\right)-n x h\left(\Delta_{F Z}\left(A_{n-2}\right), x\right) .
\end{aligned}
$$

It is known that the $h$-polynomials corresponding to $A_{n}$ and $B_{n}$ have only real zeros. Here we will show that so has $h\left(\Delta_{F Z}\left(D_{n}\right), x\right)$.

Theorem 7.1. Let $\alpha, \beta \in \mathbb{R}$ be such that $\alpha \geq 0,2 \alpha+\beta>0$ and let $n \geq 2$ be an integer. Then the polynomial

$$
F_{n}(\alpha, \beta):=\alpha h\left(\Delta_{F Z}\left(B_{n}\right), x\right)+\beta n x h\left(\Delta_{F Z}\left(A_{n-2}\right), x\right)
$$

is real- and simple-rooted. Moreover, $h\left(\Delta_{F Z}\left(B_{n-1}\right), x\right)$ strictly interlaces $F_{n}(\alpha, \beta)$ if $\alpha>0$ and strictly alternates left of $F_{n}(\alpha, \beta)$ if $\alpha=0$.

Corollary 7.2. Let $W$ be a finite Weyl group. Then $h\left(\Delta_{F Z}(W), x\right)$ has only real and simple zeros. 
Proof. For the exceptional Weyl group one can check the real-rootedness ad hoc; see [28. The other cases follows from Theorem 7.1

The Hadamard product of two polynomials

$$
\begin{aligned}
& p(x)=a_{0}+a_{1} x+\cdots+a_{m} x^{m}, \\
& q(x)=b_{0}+b_{1} x+\cdots+b_{n} x^{n}
\end{aligned}
$$

is the polynomial

$$
(p \star q)(x)=a_{0} b_{0}+a_{1} b_{1} x+\cdots+a_{N} b_{N} x^{N},
$$

where $N=\min (m, n)$. Málo proved that if the zeros of $p$ are real and the zeros of $q$ are real and of the same sign, then the zeros of $p \star q$ are real as well. This also follows from Theorem 3.8 since $p \star q=\Gamma[p S q]$, where $\Gamma$ is the multiplier sequence $\left\{\frac{1}{k !}\right\}_{k=0}^{\infty}$. It is known (see e.g. [18]) that if $f$ has only real zeros, then all zeros of $\Gamma[f]$ are real and simple except for possibly at the origin.

Proof of Theorem 7.1. We may write $F_{n}(\alpha, \beta)$ as

$$
F_{n}(\alpha, \beta)=\alpha(x+1) f+(2 \alpha+\beta) g,
$$

where $f=(x+1)^{n-1} \star(x+1)^{n-1}$ and $g=\left(x(x+1)^{n-1}\right) \star(x+1)^{n-1}$.

By the discussion before this proof we have that for all real choices of $\gamma, \delta \in \mathbb{R}$ the polynomial

$$
\gamma f+\delta g=\left((\gamma+\delta x)(x+1)^{n-1}\right) \star(x+1)^{n-1}
$$

is real- and simple-rooted. By the Obreschkoff theorem we infer that $f$ strictly alternates left of $g$. Now, since $f \preceq(x+1) f$ and $f \ll g$ we know by Lemma 2.2 that $f$ either interlaces or alternates left of $F_{n}(\alpha, \beta)$ for all $\alpha, \beta \in \mathbb{R}$ such that $\operatorname{sgn}(\alpha)=\operatorname{sgn}(2 \alpha+\beta)$. Moreover, since $g$ and $f$ have no common zeros, neither does $F_{n}(\alpha, \beta)$ and $f$ (provided that $2 \alpha+\beta \neq 0$ ).

\section{ACKNOWLEDGMENTS}

The author thanks Francesco Brenti for interesting discussions and helpful suggestions on the topic of this paper during the author's stay at Universitá di Roma "Tor Vergata". Thanks also to an anonymous referee for valuable comments on the presentation of this paper.

\section{REFERENCES}

1. M. Bóna, Corrigendum: "Symmetry and unimodality in t-stack sortable permutations", J. Combin. Theory Ser. A 99 (2002), no. 1, 191-194. MR1911466 (2003g:05007b)

2. — A survey of stack-sorting disciplines, Electron. J. Combin. 9(2) (2002). MR 2028290 (2004j:05012)

3. , Symmetry and unimodality in t-stack sortable permutations, J. Combin. Theory Ser. A 98 (2002), no. 1, 201-209. MR.1897934 (2003g:05007a)

4. M. Bousquet-Mélou, Multi-statistic enumeration of two-stack sortable permutations, Electron. J. Combin. 5 (1998), no. 1, Research Paper 21, 12 pp. (electronic). MR1614300 (99b:05001)

5. P. Brändén, On operators on polynomials preserving real-rootedness and the Neggers-Stanley conjecture, J. Algebraic Comb. 20 (2004), no. 2, 119-130. MR.2104673

6. F. Brenti, Unimodal, log-concave and Pólya frequency sequences in combinatorics, Mem. Amer. Math. Soc. 81 (1989), no. 413, viii+106. MR.0963833 (90d:05014)

7. _ Log-concave and unimodal sequences in algebra, combinatorics, and geometry: an update, Jerusalem combinatorics '93, Contemp. Math., vol. 178, Amer. Math. Soc., Providence, RI, 1994, pp. 71-89. MR.1310575 (95j:05026) 
8. - -Eulerian polynomials arising from Coxeter groups, European J. Combin. 15 (1994), no. 5, 417-441. MR1292954 (95i:05013)

9. 91 (2000), no. 1-2, 137-170. MR1779778 (2001i:05153)

10. F. Brenti, G. F. Royle, and D. G. Wagner, Location of zeros of chromatic and related polynomials of graphs, Canad. J. Math. 46 (1994), no. 1, 55-80. MR1260339 (94k:05077)

11. L. Comtet, Advanced combinatorics, enlarged ed., D. Reidel Publishing Co., Dordrecht, 1974. MR.0460128 (57:124)

12. T. Craven and G. Csordas, Multiplier sequences for fields, Illinois J. Math. 21 (1977), no. 4, 801-817. MR0568321(58:27921)

13. J. Dedieu, Obreschkoff's theorem revisited: what convex sets are contained in the set of hyperbolic polynomials?, J. Pure Appl. Algebra 81 (1992), no. 3, 269-278. MR1179101 (93g:12001)

14. S. Dulucq, S. Gire, and O. Guibert, A combinatorial proof of J. West's conjecture, Discrete Math. 187 (1998), no. 1-3, 71-96. MR1630680 (99f:05053)

15. A. Edrei, On the generating functions of totally positive sequences. II, J. Analyse Math. 2 (1952), 104-109. MR0053175 (14:732e)

16. D. Foata and M. Schützenberger, Théorie géométrique des polynômes eulériens, Lecture Notes in Mathematics, Vol. 138, Springer-Verlag, Berlin, 1970. MR0272642 (42:7523)

17. S.V. Fomin and A.V. Zelevinsky, Y-systems and generalized associahedra, Ann. of Math. 158 (2003), no. 158, 977-1018. MR2031858 (2004m:17010)

18. J. Garloff and D. G. Wagner, Hadamard products of stable polynomials are stable, J. Math. Anal. Appl. 202 (1996), no. 3, 797-809. MR1408355 (97e:30010)

19. I. P. Goulden and J. West, Raney paths and a combinatorial relationship between rooted nonseparable planar maps and two-stack-sortable permutations, J. Combin. Theory Ser. A 75 (1996), no. 2, 220-242. MR1401000 (98a:05082)

20. G. H. Hardy, J. E. Littlewood, and G. Pólya, Inequalities, University Press, Cambridge, 1952, 2d ed. MR0046395 (13:727e)

21. B. Jacquard and G. Schaeffer, A bijective census of nonseparable planar maps, J. Combin. Theory Ser. A 83 (1998), no. 1, 1-20. MR.1629428 (99f:05054)

22. S. Karlin, Total positivity. Vol. I, Stanford University Press, Stanford, Calif., 1968. MR.0230102(37:5667)

23. M. Marden, Geometry of polynomials, Second edition. Mathematical Surveys, No. 3, American Mathematical Society, Providence, R.I., 1966. MR.0225972 (37:1562)

24. N. Obreschkoff, Verteilung und Berechnung der Nullstellen reeller Polynome, VEB Deutscher Verlag der Wissenschaften, Berlin, 1963. MR0164003 (29:1302)

25. G. Pólya, Collected papers, Vol. II: Location of zeros, Edited by R. P. Boas, Mathematicians of Our Time, Vol. 8. The MIT Press, Cambridge, Mass.-London, 1974. MR58:21342

26. E. D. Rainville, Special functions, The Macmillan Co., New York, 1960. MR0107725 (21:6447)

27. V. Reiner, Descents and one-dimensional characters for classical Weyl groups, Discrete Math. 140 (1995), no. 1-3, 129-140. MR1333715 (96d:05116)

28. V. Reiner and V. Welker, On the Charney-Davis and the Neggers-Stanley conjectures, http://www.math.umn.edu/ ${ }^{\sim}$ reiner/Papers/papers.html (2002).

29. J. Schur, Zwei sätze über algebraische gleichungen mit lauter reellen wurzeln, J. Reine Angew. Math. 144 (1914), no. 2, 75-88.

30. R. P. Stanley, Log-concave and unimodal sequences in algebra, combinatorics, and geometry, Graph theory and its applications: East and West (Jinan, 1986), Ann. New York Acad. Sci., vol. 576, New York Acad. Sci., New York, 1989, pp. 500-535. MR.1110850 (92e:05124)

31. - Enumerative combinatorics. Vol. 2, Cambridge Studies in Advanced Mathematics, vol. 62, Cambridge University Press, Cambridge, 1999. MR.1676282 (2000k:05026)

32. _ Positivity problems and conjectures in algebraic combinatorics, Mathematics: frontiers and perspectives, Amer. Math. Soc., Providence, RI, 2000, pp. 295-319. MR1754784 (2001f:05001)

33. J. R. Stembridge, Some permutation representations of Weyl groups associated with the cohomology of toric varieties, Adv. Math. 106 (1994), no. 2, 244-301. MR1279220 (95f:20011)

34. R. A. Sulanke, The Narayana distribution, J. Statist. Plann. Inference 101 (2002), no. 12, 311-326, Special issue on lattice path combinatorics and applications (Vienna, 1998). MR 1878867 
35. D. G. Wagner, The partition polynomial of a finite set system, J. Combin. Theory Ser. A 56 (1991), no. 1, 138-159. MR1082848 (92b:05005)

36. Enumeration of functions from posets to chains, European J. Combin. 13 (1992), no. 4, 313-324. MR1179527 (94c:05008)

37. _ Total positivity of Hadamard products, J. Math. Anal. Appl. 163 (1992), no. 2, 459483. MR:1145841 (93f:15020)

Matematik, Chalmers tekniska hÖGskola och Göteborgs universitet, S-412 96 GöteBORG, SWEDEN

E-mail address: branden@math.chalmers.se

Current address: Department of Mathematics, University of Michigan, Ann Arbor, Michigan 48109-1043

E-mail address: branden@umich.edu 